\title{
A New WSNs Localization Based on Improved Fruit Flies Optimization Algorithm
}

\author{
Haiyun Wang \\ College of Mobile Telecommunications, Chongqing University of Posts and Telecommunications, \\ Chongqing, 401520, China
}

13340333092@163.com

Keywords: wireless sensor networks, node localization, fruit fly optimization algorithm, intelligent algorithm.

\begin{abstract}
A new node localization approach of Wireless Sensor Networks based on improved fruit fly optimization algorithm is presented to optimize the location accuracy. This approach reduce the distance error between the unknown node and beacon node by improved fruit fly optimization algorithm. Then, instruct the influence of different initial position, different population group and different number of beacons on the localization accuracy. Simulation results indicate that the localization algorithm is simple, fast convergence and high accuracy. Compared the localization accuracy that is optimized by improved APIT and improved PSO with improved fruit fly optimization algorithm, the algorithm based on improved fruit fly optimization algorithm is more accurate than other intelligent algorithm in WSNs node localization.
\end{abstract}

\section{Introduction}

Wireless sensor networks (WSNs) are systems of small, low powered networked sensing devices deployed over an area of interest to monitor interesting events and perform application specific tasks in response to them ${ }^{[1]}$. It is used in environment monitoring, military defense, industrial and agricultural control, ect. However, military or environmental monitoring are not only need monitoring information but also need location information. For a sensor, determining its location information has become an attractive research issue in WSNs. Localization algorithms are divided into two broad categories according to the type of information used: range-free and range-based ${ }^{[2-5]}$. In this paper, we use the range-base localization scheme to locate the unknown nodes.

The sections of this paper is organized as follows: In this section, recommend some background information of localization algorithms, then introduce the purpose and significance of this paper; In section 2, distance measurement method and calculation method of node coordinate will be introduced; Section 3 will introduce node localization approach based on improved FOA; Section 4 will work out the experimental simulation and analyses the result of localization; This paper is concluded in section 5 .

\section{WSNs Positioning Technology}

Through studying the range-based localization algorithm, it can be classified into distance measurements and calculating the coordinates. First of all, calculate the distance between nodes by range-based localization algorithm. Then calculate the coordinates of the unknown nodes by node coordinate calculation method.

\subsection{The calculation method of node coordinates}

Trilateral measuring method is kind of location algorithm which is easy to realize and its cost is little in localization algorithm. The advantage of trilateral measurement method is that just need to know the coordinate of three beacon nodes. Calculation is simple, but the distance between unknown nodes to the three beacon nodes is not accurate in the practical application due to measurement errors. Such as $\mathrm{A}, \mathrm{B}, \mathrm{C}$ are known location beacon nodes. The unknown node should be located in the circle with $\mathrm{A}$ as 
the center. The same node should be located in the circle with B, C as the center as shown in Fig. 1(a). In theory, the three round of intersection is the location of the unknown node. However, the three round does not intersect the circle at one point in actual positioning system as shown Fig. 1(b), (c). Therefore, it needs to optimize the distance error by optimization algorithm, reducing the shaded area.
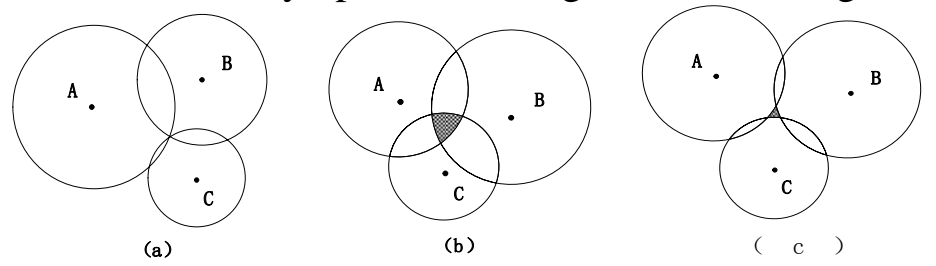

Fig.1 The case of Trilateration graph

If there are multiple beacon nodes as: $\left(x_{1}, y_{1}\right),\left(x_{2}, y_{2}\right),\left(x_{3}, y_{3}\right), \cdots,\left(x_{i}, y_{i}\right), \cdots,\left(x_{n}, y_{n}\right)$.Each beacon node to the unknown node distance is $r_{1}, r_{2}, \ldots, r_{i}, \ldots, r_{n}$. The relationship between the unknown node and beacon nodes are :

$$
\varepsilon_{i}=\left|\sqrt{\left(\hat{x}-x_{i}\right)^{2}+\left(\hat{y}-y_{i}\right)^{2}}-r_{i}\right|, i=1,2,3, \cdots, n
$$

Where $(\hat{x}, \hat{y})$ is the estimated position of unknown node. $\varepsilon_{\mathrm{i}}$ are error which between estimation distance and actual distance. Therefore, the sum of the measured error between the node and the beacon nodes can be expressed as:

$$
g(\hat{x}, \hat{y})=\sum_{i=1}^{n} \varepsilon_{i},(n \geq 3)
$$

When $g(\hat{x}, \hat{y})$ take the minimum value, the value of $(\hat{x}, \hat{y})$ is closest to the actual value.

\section{Node Localization Approach Based on Improved FOA}

\subsection{Fruit Fly optimization algorithm}

The Fruit Fly Optimization Algorithm (FOA) is a new method for finding global optimization based on the food finding behavior of the fruit fly. Based on the food finding characteristics of the fruit fly, it is divided into several necessary steps. The steps areas follows ${ }^{[6]}$ :

Step 1: Random initial fruit fly group location (IntX_axis,IntY_axis), determine the number of group sizepop and the maximum iterations maxgen.

Step 2: Give the random direction for the search of food using osphresis by an individual fruit fly.

$X_{i}=X_{-}$asix + Randvalue $\quad Y_{i}=Y_{-}$asix + Randvalue

Step 3: Estimating the distance (Dist $\left.{ }_{i}\right)$ between fruit fly and the origin, then calculate the smell concentration judgment value $\left(S_{i}\right)$.

$$
\text { Dist }_{i}=\sqrt{X_{i}^{2}+Y_{i}^{2}}, S_{i}=1 / \text { Dist }_{i}
$$

Step 4: Substitute smell concentration judgment value ( $\mathrm{Si}$ ) into smell concentration judgment function so as to find the smell concentration $\left(\right.$ Smell $\left._{i}\right)$.

Smell $_{i}=$ Function $\left._{\left(S_{i}\right.}\right)$

Step 5: Find out the fruit fly with maximal smell concentration among the fruit fly group.

[bestSmell bestIndex] $=\max \left(\right.$ Smell $\left._{i}\right)$

Step 6: Keep iterative best smell concentration value and $x, y$ coordinate, and at this moment, the fruit fly swarm will use vision to fly towards that location.

Smellbest $=$ bestSmell $, x=X($ bestIndex $), y=Y($ bestIndex $)$

Enter iterative optimization to repeat the implementation of step 2-5, then judge if smell concentration is superior to the previous iterative smell concentration, if so, implement step 6. 


\subsection{Node localization based on improved FOA}

This algorithm will obtain the unknown node coordinate by reducing the measured distance error between nodes and beacon nodes.For the trilateral measurement method, we use three beacon nodes to locate unknown nodes. Where $\left(x_{\mathrm{A}}, y_{\mathrm{A}}\right),\left(x_{\mathrm{B}}, y_{\mathrm{B}}\right),\left(x_{\mathrm{C}}, y_{\mathrm{C}}\right)$ represent the coordinate of three beacon nodes. The error function is shown as (4):

$$
\begin{aligned}
& \delta=\frac{1}{3} g(\hat{x}, \hat{y})=\frac{1}{3} \sum_{i=1}^{3} \varepsilon_{i} \\
& =\frac{1}{3}\left(\left|\sqrt{\left(\hat{x}-x_{A}\right)^{2}+\left(\hat{y}-y_{A}\right)^{2}}-r_{A}\right|+\left|\sqrt{\left(\hat{x}-x_{B}\right)^{2}+\left(\hat{y}-y_{B}\right)^{2}}-r_{B}\right|+\left|\sqrt{\left(\hat{x}-x_{C}\right)^{2}+\left(\hat{y}-y_{C}\right)^{2}}-r_{C}\right|\right)
\end{aligned}
$$

When $\delta$ taking the minimum, it means that unknown node $(\hat{x}, \hat{y})$ close to the actual measure value. $\mathrm{r}_{\mathrm{A}}, \mathrm{r}_{\mathrm{B}}, \mathrm{r}_{\mathrm{C}}$ is the distance between beacon node to unknown node. In order to improve the positioning accuracy, we can use improved fruit fly algorithm to reduce the error.

Set up initial location of fruit fly flying range is $[0,5]$ randomly, the random fly direction and distance zone for fruit fly searching food is $[-0.5,0.5]$, fruit fly population size is sizepop $=30$, and iterative number is maxgen $=100$. Estimate the distance between fruit fly and the origin point $D(i)$, that is the distance between unknown node and beacons, as (5):

$$
D(i)=\left|\sqrt{\left(\hat{x}-x_{i}\right)^{2}+\left(\hat{y}-y_{i}\right)^{2}}-r_{i}\right|
$$

Substitute smell concentration judgment value $D(i)$ into smell concentration judgment function:

$$
\operatorname{Smell}(i)=\frac{1}{3} \sum_{i=1}^{3} D(i)
$$

Find the largest smell concentration of fruit flies in order to make the smallest error sum of squares. Retain the best $(X(i), Y(i))$ coordinates. Enter iterative optimization to repeat the implementation of steps $2-5$, then judge if the smell concentration is superior to the previous iterative smell concentration, if so, implement step 6.

\section{Simulation and Performance Analysis}

For validating the performance of improved fruit fly algorithm, this paper implements a series of simulation for this localization algorithm. Assume the $\mathrm{P}(3,2)$ is coordinates of the unknown node. Three beacon nodes coordinates are $\mathrm{A}(0,0), \mathrm{B}(5,0), \mathrm{C}(0,5)$. It can calculate the $\mathrm{P}$ to $\mathrm{A}, \mathrm{B}, \mathrm{C}$ distance are $r_{1}=\sqrt{13}, r_{2}=\sqrt{8} 、 r_{3}=3 \sqrt{2}$. In the initial parameter set up of the fruit fly population size is 30 , and iterative number is 100 . Analyze the influence of different initial position, initial population, the number of beacon nodes and communication distance on the positioning accuracy. Compared with the relative error and standard deviation of node localization of WSNs based on improved APIT algorithm (APIT) ${ }^{[7]}$ and improved particle swarm optimization (PSO) ${ }^{[8]}$, the accuracy of improved fruit fly optimization algorithm(FOA) is more accurate than other intelligent algorithm in wireless sensor network node localization.

\subsection{The improved fruit flies algorithm convergence}

After optimization by the fruit flies algorithm, the fruit flies focus on $(3,2)$ as shown in the Fig. 2. Due to the increased number of iterations, the unknown node error decrease. After seven iterations, the mean square error almost close to zero in Fig.3. After 100 times of iterative evolution, meet the requirements of the positioning error with a coordinate of $(3.0043,1.9961)$. It is very close to actual coordinates of the point $\mathrm{P}(3,2)$. It also shows that the fruit flies optimization algorithm convergence speed is quick. 


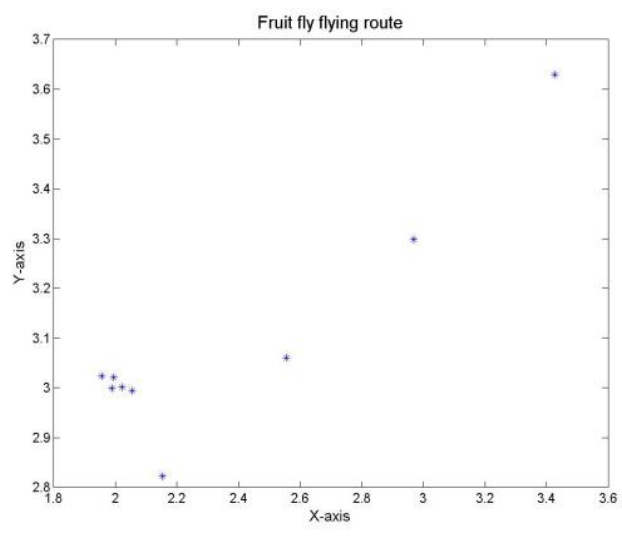

Fig.2 Fruit Fly Flying Route

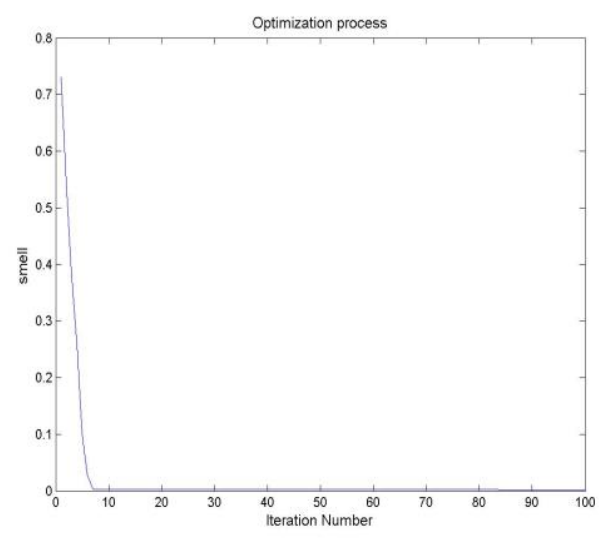

Fig.3 Optimization Process of unknown node

\subsection{The influence of initial position and initial population on positioning}

In the initial parameter set up of the fruit fly, the random initialization fruit fly swarm location range is $[0,5]$, the random fly direction and distance zone of iterative fruit fly food searching is $[-0.5,0.5]$, fruit fly population size is sizepop $=30$, and iterative number is maxgen $=100$. Setting the unknown node is $(2,3)$. The first line of two graphs in Fig. 4(a) is the iterative and the flight path of fruit flies.

(a) Different initial position and optimization process

The random initialization fruit fly swarm location range is $[0,30]$. The second line of two graphs in Fig.4(a) is iterative and flight path figure which is changed initial fruit position. Because of changed initial position, it finds the final fruit flies gather points diffically.

(b) Different population group and optimization process

Choose a different population size sizepop $=10$.The second line of two graphs in Fig.4(b) is iterative and flight path figure which is changed population size of fruit flies. After change the population size, the fruit flies flight path is not clear and the convergence speed is slowed.

(c) Different initial position, population group and optimization process

The random initialization fruit fly swarm location range is $[0,30]$, fruit fly population size is sizepop $=50$. The second line of two graphs in Fig.4(c) is iterative and flight path figure which are changed population size and changed initial of fruit flies. After change the population size and initial, it is not easy to find the final fruit flies gather points and slow the convergence speed.

After testing shows that choose the appropriate number of iterations, population size, initial position and flying range are very important for localization.
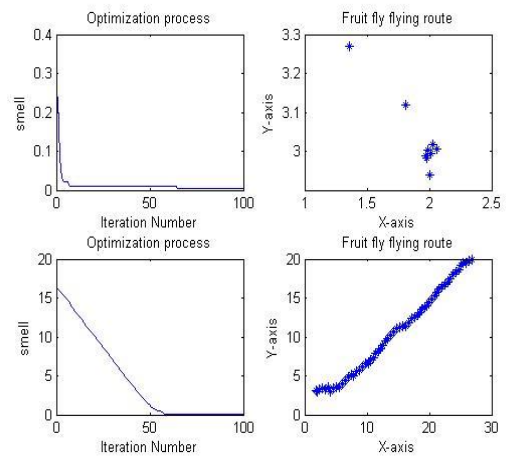

(a)

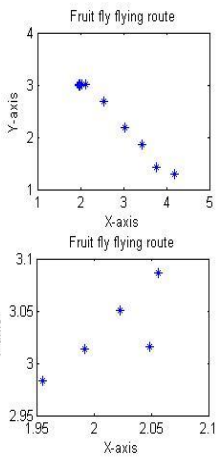

(b)
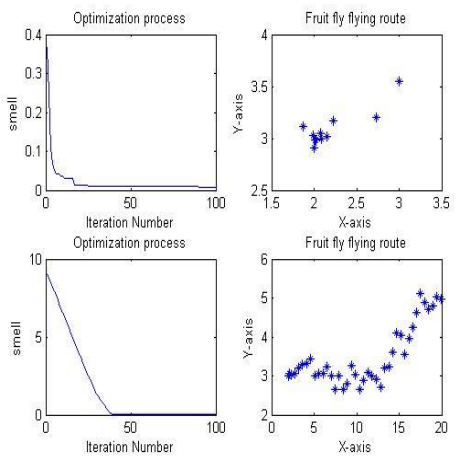

(c)

\subsection{Improved FOA compared with other intelligent algorithms}

As shown in table 1, compared with the relative error and standard deviation of improved APIT and improved PSO, the accuracy of improved FOA is more accurate than other intelligent algorithm in WSNs node localization. 
Table 1 Comparing Improved FOA with Improved APIT and Improved PSO in localization

\begin{tabular}{|c|c|c|c|c|c|c|}
\hline \multirow[b]{2}{*}{$\begin{array}{c}\text { Actual } \\
\text { coordinate } / \mathrm{m}\end{array}$} & \multicolumn{2}{|c|}{ improved APIT } & \multicolumn{2}{|c|}{ Improved PSO } & \multicolumn{2}{|c|}{ Improved FOA } \\
\hline & $\begin{array}{c}\text { measured } \\
\text { coordinate } / \mathrm{m}\end{array}$ & $\begin{array}{l}\text { relative } \\
\text { error }\end{array}$ & $\begin{array}{c}\text { measured } \\
\text { coordinate } / \mathrm{m}\end{array}$ & $\begin{array}{l}\text { relative } \\
\text { error }\end{array}$ & $\begin{array}{c}\text { measured } \\
\text { coordinate } \\
\text { /m }\end{array}$ & $\begin{array}{l}\text { relative } \\
\text { error }\end{array}$ \\
\hline$(0.4,3.0)$ & $(0.400,2.960)$ & $1.33 \%$ & $(0.399,2.889)$ & $0.24 \%$ & $\begin{array}{c}(0.399, \\
2.996)\end{array}$ & $0.15 \%$ \\
\hline$(2.5,3.2)$ & $(2.490,3.210)$ & $0.36 \%$ & $(2.491,3.188)$ & $0.37 \%$ & $\begin{array}{c}(2.496, \\
3.203)\end{array}$ & $0.11 \%$ \\
\hline$(1.2,2.4)$ & $(1.200,2.390)$ & $0.42 \%$ & $(1.199,2.389)$ & $0.27 \%$ & $\begin{array}{c}(1.200, \\
2.396)\end{array}$ & $0.17 \%$ \\
\hline$(2.5,1.4)$ & $(2.470,1.370)$ & $1.67 \%$ & $(2.510,1.399)$ & $0.24 \%$ & $\begin{array}{c}(2.518, \\
1.399) \\
\end{array}$ & $0.16 \%$ \\
\hline$(3.6,3.5)$ & $(3.600,3.470)$ & $0.86 \%$ & $(3.588,3.498)$ & $0.17 \%$ & $\begin{array}{c}(3.600, \\
3.505)\end{array}$ & $0.09 \%$ \\
\hline $\begin{array}{l}\text { standard } \\
\text { deviation }\end{array}$ & \multicolumn{2}{|c|}{$1.18 \%$} & \multicolumn{2}{|c|}{$0.30 \%$} & \multicolumn{2}{|c|}{$0.17 \%$} \\
\hline
\end{tabular}

\section{Conclusion}

This paper propose a new node localization approach based on improved FOA. The approach can reduce the distance error between unknown node and beacon node to improve accuracy. Instruct the influence of different initial position, different population group and different number of beacons on the localization accuracy. By simulation results indicate that the localization algorithm is simple, fast convergence and high accuracy. Compared with the relative error and standard deviation of improved APIT and improved PSO, the accuracy of improved FOA is more accurate than other intelligent algorithm in WSNs node localization.

\section{References}

[1] Xiao Chen, Neil C. Rowe, Jie Wu, ect. Improving the localization accuracy of targets by using their spatial-temporal relationships in wireless sensor networks. J. Parallel Distrib. Comput. 72 (2012). pp: 1008-1018

[2] Mohammad Shaifur Rahman, Youngil Park, Ki-Doo Kim. RSS-Based Indoor Localization Algorithm for Wireless Sensor Network Using Generalized Regression Neural Network. Arabian Journal for Science and Engineering Volume 37, Issue 4. pp: 1043-1053

[3] Zhonghua HUANG, Shunhua LI. The Study on WSN Node Positioning Algorithm Improvement [C]// 2012 International Conference on Computer Science and Service System. Nanjing, China. IEEE Press, 2012. pp: 1248-1249.

[4] Anil K, Arun K, Jasbir S S, etal. Computational Intelligence Based Algorithm for Node Localization in Wireless Sensor Networks[C]//2012 $6^{\text {th }}$ IEEE International Conference Intelligent Systems. Sofia, Bulgaria. IEEE Press, 2012. pp: 431 - 438

[5] Lihong ZHANG. Wireless Sensor Network Localization Based on Improved DV-Hop Algorithms [J]. Microelectronics \& Computer. 2012, 29(9). pp: 171-174

[6] Pan W T. A new fruit fly optimization algorithm: taking the financial distress model as an example [J]. Knowledge-Based Systems. 2011, 26. pp: 69-74

[7] Tianhong DAI, Hao LI. Node localization of wireless sensor networks based on improved APIT algorithm [J]. Transducer and Microsystem Technology 2016, 1(35) PP: 135-138

[8] Jianfeng REN, Yongqiang ZHANG. Integrated Network Intelligent Node Deployment Algorithm of Improved [J]. Computer Science. 2013, 3(40) . pp: 183-186 